Intersections

Canadian Journal of Music

Revue canadienne de musique
Intersections CANADIAN JOURAL OF MUSIC
REVUE CANADIENEE DE MUSIOUH

\title{
Les enjeux de la cartographie sonore par rapport à la frontière de la neutralité
}

\section{Rainier Leloup}

Volume 37, numéro 2, 2017

URI : https://id.erudit.org/iderudit/1066618ar

DOI : https://doi.org/10.7202/1066618ar

Aller au sommaire du numéro

\section{Éditeur(s)}

Canadian University Music Society / Société de musique des universités canadiennes

\section{ISSN}

1911-0146 (imprimé)

1918-512X (numérique)

Découvrir la revue

Citer cet article

Leloup, R. (2017). Les enjeux de la cartographie sonore par rapport à la frontière de la neutralité. Intersections, 37(2), 59-72.

https://doi.org/10.7202/1066618ar
Résumé de l'article

Les cartographies sonores émergent et se développent de plus en plus depuis les années 2000. À l'origine, il ne s'agissait que d'une simple représentation sonique d'un lieu, mais progressivement ces interfaces numériques ont basculées de " cartes » à " contre-cartes » par l'engagement social, culturel ou politique de leurs créateurs. Outre des ambiances sonores, ces " contre-cartes " révèlent des points de vue particuliers sur l'environnement quotidien d'un individu, sans se soumettre à un contrôle d'une quelconque autorité. Cet article observe les enjeux de ces créations à travers plusieurs exemples et propose un modèle d'analyse des indices topographiques d'un enregistrement sonore.
Copyright ( C Canadian University Music Society / Société de musique des universités canadiennes, 2019
Ce document est protégé par la loi sur le droit d'auteur. L'utilisation des services d'Érudit (y compris la reproduction) est assujettie à sa politique d'utilisation que vous pouvez consulter en ligne.

https://apropos.erudit.org/fr/usagers/politique-dutilisation/ 


\title{
LES ENJEUX DE LA CARTOGRAPHIE SONORE PAR RAPPORT À LA FRONTIĖRE DE LA NEUTRALITÉ
}

\author{
Rainier Leloup
}

En 1972, le film de Blaxploitation «Across the $110^{\text {th }}$ street» a été projeté dans les cinémas new-yorkais. La chanson principale (du même nom que le film) fut composée par le chanteur soul noir américain Bobby Womack. Dans ce morceau, Womack insiste sur la vie difficile du ghetto new-yorkais: "Harlem est la capitale de toutes les villes ghettoïsées ${ }^{1} »$. Ce moment précis dans les paroles de ce morceau, au style et au genre particulier dans l'histoire de la musique populaire américaine, révèle clairement la connexion entre la musique et l'environnement géographique. Dans l'entièreté du morceau, Bobby Womack ne cesse de décrire la vie quotidienne misérable d'un ghetto noir américain des années 1970, pour enfin bien préciser l'endroit où ce ghetto commence, à savoir au-dessus de Central Park à New York, une fois traversée la $110^{\mathrm{e}}$ rue. En plus d'une description musicale d'un lieu, le référent topographique est appuyé par le film, qui met en scène deux détectives noirs américains enquêtant dans ce même quartier. En réalité, tout enregistrement sonore recèle d'indices topographiques. Sur une carte, ces morceaux offrent un point de vue différent d'une réalité parfois impossible à exprimer autrement qu'à travers une création de type culturelle. Cette perception particulière d'un territoire ne convient pas toujours aux responsables de la représentation de ce même territoire. Si c'est le cas pour les descriptions musicales du ghetto de Harlem par Bobby Womack en 1972, c'est aussi le cas, de manière plus contemporaine, des cartographies sonores.

\section{EN QUELQUES MOTS}

Qu'est-ce qu'une cartographie sonore? La cartographie sonore est un médium qui émerge principalement dans les années 2000 (Waldock 2011), sur la base théorique des travaux fondateurs de Raymond Murray Schafer dans les années 1970. Il s'agit d'une interface numérique proposant une représentation spatialisée du son, quelle que soit sa forme. Cette spatialisation peut être totalement abstraite ou alors s'apparenter à une carte citadine. Dans ce dernier cas, on dira qu'il s'agira alors d'une représentation sonique d'une localisation

1 «Harlem is the capital of every ghetto town.» (traduction par l'auteur). 
spécifique. En 1977, Schafer dévoile son travail sous le nom de "paysage sonore» (Schafer 2010) dans lequel son objectif principal fut de conscientiser l'individu de son environnement sonore, débordant sur une interprétation politique de la modernisation américaine basée sur le modèle postfordiste. Selon ses propres termes: "Le paysage sonore se définit comme champ d'étude acoustique, quel qu'il soit. Ce peut être une composition musicale, un programme de radio ou un environnement acoustique. On isole et on étudie un environnement acoustique comme on analyse les caractéristiques d'un paysage donné» (Schafer 2010, 29). Pour Schafer, les sons hi-fi se rapportent aux champs, aux pâturages et à la nature en général dans sa forme la plus isolée de l'implication humaine. En revanche, les sons $l o-f i$ se rapportent au cour d'une ville dans laquelle le silence n'existe pas. Ce qui fait la différence dans ces deux types d'enregistrements est la distinction totale ou impossible d'un son unique. Le bruit du vent dans un champ de blé se reconnaîtra alors que celui d'une voiture parmi une vingtaine d'autres sera moins perceptible. L'engagement politique d'une telle démarche est manifeste et qualifié de démocratique selon Schafer.

Cet engagement politique fut critiqué trente ans plus tard par Andra McCartney, dénonçant l'aspect détourné de cette quête d'authenticité du son «pur» (McCartney 2010). Qui plus est, la volonté démocratique perd de sa verve si l'on questionne l'accès aux données issues de la recherche. En effet, les enregistrements ne replaçaient pas tous les individus dans leur environnement sonore, mais bien plutôt ceux qui pouvaient se permettre de s'intéresser à une telle recherche. Enfin, Jacqueline Waldock met également en lumière l'importance du sexe de la personne qui enregistre le son, rappelant que l'ambiance sonore sera différente (en ville particulièrement) selon que cette personne soit une femme ou un homme. Dès lors, la prise de son ne peut être considérée comme donnée sonique unique d'une localisation spécifique (Waldock 2011).

Cet objectif de conscientisation de l'espace environnemental n'est pas sans précédent. La seconde moitié du $\mathrm{XX}^{\mathrm{e}}$ siècle est marquée par une critique à tout niveau à propos de la représentation territoriale. Toute carte est un objet politique qui échappe au contrôle de l'individu qui justement habite un même territoire. Foucault illustre cette relation par le Panopticon de Bentham (1975). Dans cet édifice carcéral, il y a, selon lui, un individu disciplinaire (le gardien) qui surveille les individus disciplinés (les prisonniers) du haut d'une tour centrale à l'intérieur de la prison. L'individu discipliné est libre de toute navigation dans l'enceinte du bâtiment, seulement tous ses moindres mouvements sont contrôlés par l'individu disciplinaire. Par-là, Foucault distingue en réalité deux points de vue qui sont coutumiers de l'urbaniste: le point de vue intérieur (celui de l'habitant), et le point de vue extérieur (celui de l'autorité). Cette relation est analogue au travail cartographique pour lequel l'état doit s'assurer de la représentation de son territoire. En d'autres termes (Branch 2014, 165):

La profonde connexion entre les images cartographiées et l'autorité politique n'est absolument pas un phénomène historique exceptionnel. [...] lors de la fin du XXème siècle, les états ont continué d'essayer de contrôler la manière dont leurs territoires fut cartographié — avec les cartes «incorrectes» perçues comme une menace envers l'identité propre de la nation. 
Ces efforts pour contrôler la cartographie se sont étendus depuis les modalités de la carte au contrôle de la personne responsable de cartographier le territoire de l'état. Cette dernière problématique, en réalité, est probablement devenue de plus en plus importante pour les gouvernements, étant donné la croissance de créations et distributions cartographiques qui échappent à leur mainmise ${ }^{2}$.

À travers ce commentaire, l'auteur Jordan Branch mentionne cette critique à tous niveaux à propos de la représentation territoriale qui a pris le nom de «contre-carte» (Presner, Shepard, et Kawano 2014). Cette production de «contre-cartes» a pour objectif une réappropriation du territoire par l'habitant, ce que de Certeau écrit en ces termes $(1990,57)$ :

Producteurs méconnus, poètes de leurs affaires, inventeurs de sentiers dans les jungles de la rationalité fonctionnaliste, les consommateurs produisent quelque chose qui a la figure des "lignes d'erre" dont parle Deligny. Ils tracent des «trajectoires indéterminées» apparemment insensées parce qu'elles ne sont pas cohérentes avec l'espace bâti, écrit et préfabriqué où elles se déplacent.

En effet, sur le plan scientifique, cet objectif a été mis en lumière par de nombreux auteurs (pour n'en citer que quelques-uns: Debord 1955; Deleuze et Guattari 1980; Foucault 1990; Lefebvre 2000). Gilles Deleuze et Félix Guattari (1980) élaborent le concept de la ritournelle, un code sonore qui délimiterait la zone de confort d'un être humain, sa bulle, voire son territoire. À travers la ritournelle, Deleuze et Guattari replacent l'être humain, les modalités de son processus de territorialisation, au centre de l'expérience qui l'unit au temps et au lieu. Dans un autre ordre d'idées, le philosophe Guy Debord (1955) propose des exercices de ce qu'il appelle "psychogéographie» en insistant sur la perception personnelle du plan urbain. Ces exercices brisent "l'espace bâti et préfabriqué » dont parle Michel de Certeau et pousse l'individu à avancer tout droit sur sa trajectoire, par exemple, dans la ville sans se préoccuper des interdits autoritaires sur lesquels son pouvoir est limité (tel qu'une propriété privée, un canal ou un immeuble de services publics).

Sur le plan culturel, de nombreux travaux comme ceux des artistes Anish Kapoor, Christo, Joseph Beuys, Bouchra Khalili, ou encore Orson Welles développent une perspective alternative sur la perception de l'environnement géographique. Dans son œuvre «Cloud Gate» (2006), l'artiste indo-britannique Anish Kapoor impose une masse gigantesque sous forme de miroir à un seul côté en plein milieu d'une mégapole. Cette bulle reflète ses alentours de manière à passer chromatiquement inaperçue dans son environnement, pourtant

2 «The deep connection between mapped images and political authority [...] is by no means an exclusively historical phenomenon. [...], in the late twenieth century, states continued to attempt to assert control over how their territories were mapped - with «incorrect» maps seen as a threat to the very identity of the nation. These efforts to control cartography extend from issues of map content to attempts to dictate who is allowed to map the state's territory. The latter issue, in fact, is probably becoming increasingly important to governments, thanks to the ways in which map creation and distribution are escaping their grasp.» (traduction par l'auteur). 
par sa surface courbée, elle propose au spectateur un environnement détourné dans lequel la ligne droite maîtresse dessinée par la ligne d'horizon n'a plus sa place. Face à cette œuvre, le passant est plongé entre deux environnements différents, l'un droit, l'autre rond (Krone et Von Stosch 2008). Christo (ainsi que d'autres artistes du mouvement du Land Art), insiste quant à lui sur l'importance du pèlerinage pour accéder à une œuvre en pleine nature. Durant le trajet pour y parvenir, le spectateur est souvent amené à réfléchir de manière introspective sur sa place face à la grandeur naturelle qui l'entoure (Spies et Volz 1980). Pour sa part, Bouchra Khalili dessine, dans «The Mapping Journey Project» (2008-2011), les trajets récités par des réfugiés sur une carte de l'Europe et de l'Afrique du Nord. De cette manière, l'artiste exprime clairement le conflit entre l'autorité cartographique et l'expérience individuelle du territoire. Par prédilection, ce sont les arts visuels et expérimentaux (Land Art, Avantgarde, ou plus généralement encore, le cinéma) qui rendent compte le plus efficacement de cette perspective. Pourtant, bien avant, de nombreux poètes témoignaient déjà du malaise face à leur environnement urbain, dont entre autres Samuel Taylor Coleridge ou Charles Baudelaire3.

Il y a donc deux aspects à la carte, séparés par une frontière. d'un côté, le territoire représenté et contrôlé par l'autorité, que l'on constate sur l'interface Google Maps lorsque l'on zoome sur la zone du Jammu-et-Cachemire par exemple - assez représentative des problèmes liés à la cartographie politique. Et de l'autre, il y a une carte déformée, transformée, modulée qui est celle du territoire réapproprié par un individu. Le terme «neutralité» est discutable. Dans ce cas-ci, il qualifie les cartes qui n'ont d'autre objectif que leur fonctionnalité. La réappropriation de l'environnement géographique immédiat fait intervenir directement la dimension personnelle dans la carte. Dans le cas d'une ville, l'implication de cette dimension transforme cette ville en une "HyperCité », c'est-à-dire «une cité réelle, couverte par des réseaux épais d'informations qui non seulement catalysent le présent, mais retournent également dans le temps et projettent de nouvelles possibilités dans le futur. Les HyperCités sont toujours en construction ${ }^{4}$ (Presner, Shepard, et Kawano 2014, 6). Dans leur projet, les trois chercheurs superposent différents calques sur une ville, qu'ils soient autoritaires ou personnels, pour en fabriquer une «HyperCité»; le préfixe hyper- se réfère à la quantité abondante d'interprétations et de directions différentes. Sur base de fondations citadines réelles, les chercheurs y ont associé sur une plateforme numérique regroupant des témoignages personnels à propos d'une localisation spécifique au sein de la ville en question. De cette manière, la ville est découverte sous tous ses aspects.

3 Voir à ce sujet: Liinnamaa 2014 et Cooper 2015.

4 "A HyperCity is a real city overlaid with thick information networks that not only catalyze the present but also go back in time to document the past and go forward to project future possibilities. HyperCities are always under construction.» (traduction par l'auteur). 


\section{ÉLABORATION DU MODÈLE}

Comment ce contexte-ci s'applique-t-il à la musique ou par extension à l'art sonore? Grâce à la cartographie sonore qui, nous l'avons abordé tout au début, concerne la production musicale, acoustique, sonographique sous tous ses aspects. Une question plus adéquate serait alors la suivante: dans quelle mesure une cartographie sonore peut-elle se positionner vis-à-vis de la frontière de la neutralité? Quelles sont les véritables modalités qui lui permettent de se positionner?

Dans mes recherches, j'en suis arrivé à distinguer quatre couches cartographiques qui pouvaient s'appliquer à la création musicale et à deux caractéristiques fondamentales à partir des concepts élaborés par Michel de Certeau (1990) et Henri Lefebvre (2000). Ces couches cartographiques constituent l'environnement géographique d'une création musicale et s'organisent autour de cette dernière. Elles répertorient tous les indices topographiques d'un enregistrement musical ou sonore selon certains paramètres particuliers. Ensemble, elles forment un modèle méthodologique pour l'analyse de ces indices. Dans L'invention du quotidien 1. Arts de faire, Michel de Certeau oppose la carte et le parcours (de Certeau 1990, 172-176). La carte se rapporterait à une description scrupuleuse d'un lieu alors que le parcours mettrait en scène un mouvement dans cette description. La différence provient principalement du discours, le parcours est organisé autour d'un pronom personnel alors que la carte n'en est pas disposée. Dans le même ordre d'idée, il oppose également les concepts d'espace et de lieu. Le second se rapporte à un espace figé, construit et organisé de manière linéaire alors que le premier met en avant les rencontres dynamiques dues aux différents mouvements au sein d'une organisation chaotique. Tout comme chez Foucault, la première opposition vient de l'insertion de l'individu sur le plan urbain et la seconde, de son activité. Henri Lefèbvre oppose, lui, ce qu'il nomme l'espace de représentation et la représentation de l'espace. Le premier se rapporte à l'imagerie et les récits mythiques associés à un lieu, voire la représentation mentale personnelle qu'un individu se fait d'un environnement bien précis (Lefèbvre 2000, 266). À la différence, la représentation de l'espace concerne plutôt l'agencement du plan urbain par une instance particulière. Ici aussi, les deux ordres que sont celui de l'individu et celui de l'autorité sont en conflit. C'est sur ces oppositions que les quatre couches cartographiques qui constituent le modèle méthodologique s'organisent.

\section{Couche cartographique abstraite}

Elle englobe tous les indices se rapportant à l'espace de représentation (Lefebvre 2000) d'un individu par rapport à son environnement. Qu'il s'agisse du contexte social, politique, idéologique, économique ou culturel ou bien des sensations de l'utilisateur, cette couche est constituée majoritairement de symboles. De manière illustrée, un citadin percevra la campagne d'une manière différente que celle du fermier (pour reprendre la formule à Henri Lefèbvre). 


\section{Couche cartographique concrète}

Elle concerne le mode de transmission des indices cartographiques d'une création musicale: le son, principalement. Dans cette couche, il est question de spatialisation du son, d'un repérage des distances dans un enregistrement entre l'auditeur et la source du son, et des stratégies spécifiques liées à ces indices topographiques.

\section{Couche cartographique théorique}

Elle situe la création musicale sur un plan théorique, constitué de différents réseaux, chronotopes et lignes. Il s'agit dans cette couche de repérer les plans impersonnels, dont les informations sont validées par la collectivité, dans lesquels la création musicale peut naviguer.

\section{Couche cartographique empirique}

À la lumière des informations de la couche cartographique théorique, cette dernière dessine véritablement le trajet de la création musicale. Dans le cas d'une cartographie sonore, l'utilisateur dessine ainsi sa carte.

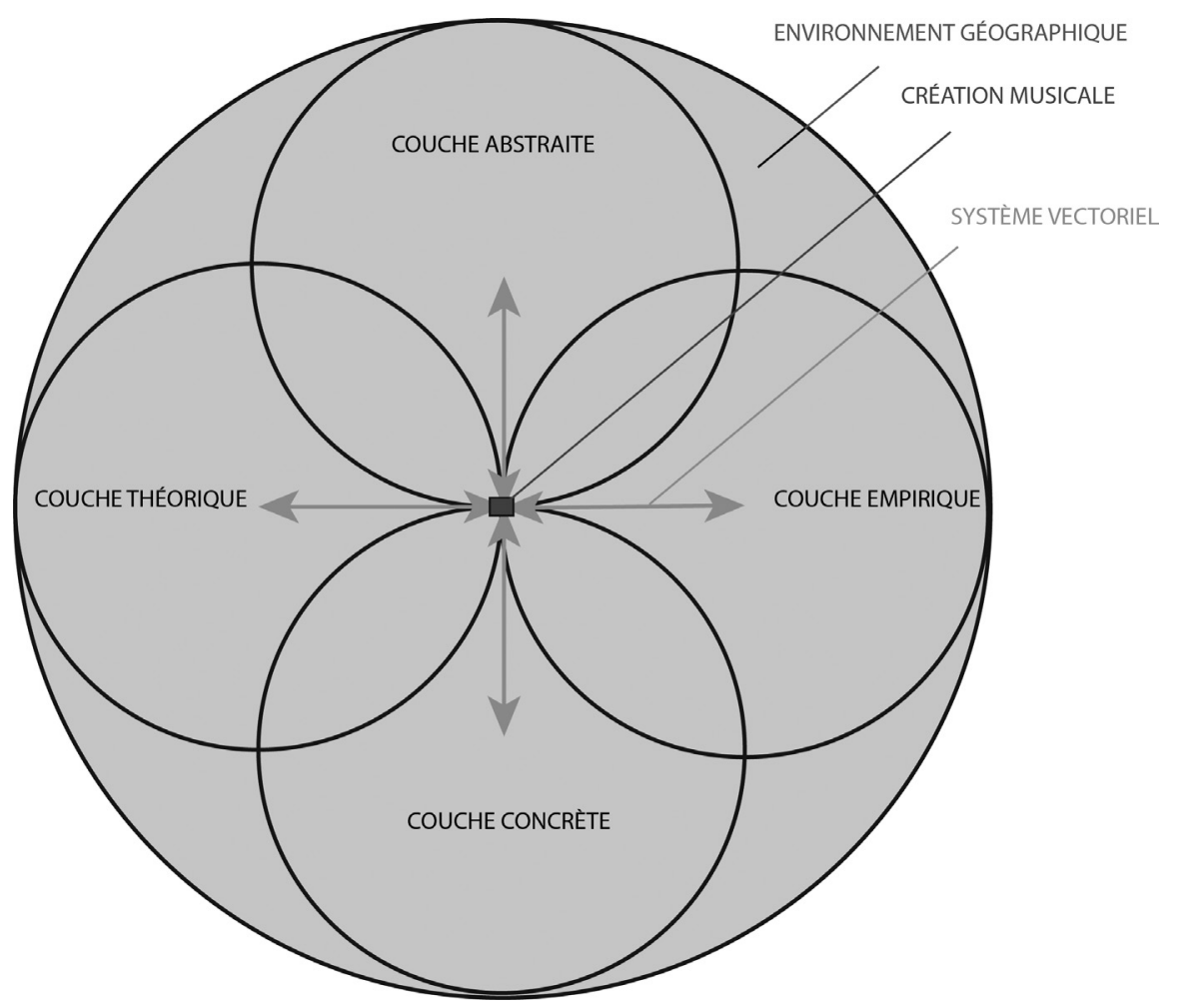

Figure 1. Schéma du modèle méthodologique pour l'analyse des indices topographiques dans l'enregistrement sonique. 
À titre d'exemple d'utilisation de ce modèle, utilisons le morceau «The Ghetto", enregistré en 1970 par le musicien soul noir américain Donny Hathaway. Dans la couche cartographique abstraite seront répertoriés les indices liés à l'imagerie du ghetto. Les référents dans le morceau et son contexte qui génèrent une suspicion et une fascination de la part d'un habitant à l'égard de l'idole pour le simple fait d'avoir réussi à s'évader des murs invisibles dont parlait le sociologue Kenneth B. Clark $(1989,36)$. Dans "The Ghetto», Donny Hathaway embellit l'image du ghetto (selon la chroniqueuse Dyanna Williams; dans Payne 2008), qui fait alors partie constituante de l'identité noire américaine, contrastant avec la description misérabiliste des sociologues de l'époque5. Se retrouvent également dans cette couche les référents aux différentes idéologies noir américaines de l'époque, qu'elles soient sociales (panafricanisme et particularisme ethnique), politiques (le «Pouvoir de l'Amour» et les Panthères Noires) ou sociétales comme le statut de la créativité au sein du ghetto ou son cosmopolitisme.

Dans la couche cartographique concrète se retrouve la stratégie sonore opérée par Donny Hathaway pour procurer à l'auditeur un certain sentiment particulier. L'enregistrement fait circuler d'un écouteur à un autre différents instruments et place les volumes et les intensités sonores à différents endroits particuliers dans l'espace sonique. De cette manière, l'auditeur se retrouve inclus dans une activité de groupe à laquelle il participe en dansant ou tapant des mains (selon que la version du morceau soit live ou en studio). Par ailleurs, outre le fait d'être placé au milieu du groupe, les distances enregistrées (un cri lointain, une note au clavier extrêmement proche) donnent forme à la profondeur du groupe. L'auditeur est inclus et actif dans une expérience de communauté.

Dans la couche cartographique théorique, le morceau est replacé sur une carte dont les informations sont généralement admises par la collectivité. Il s'agit ici de définir le ghetto selon les termes d'époque et de situer le morceau en utilisant un système particulier. Les éléments constituants le ghetto théorique dans la définition deviennent des référents dans les paroles du morceau, comme l'importance de la drogue, la place de l'église ou la dislocation familiale. En revanche, la couche cartographique empirique dessine la trajectoire du morceau selon le système adopté par celui qui construit cette cartographie (par exemple, depuis la parution du morceau jusqu'à son écoute la plus récente).

Organisés un à un et répertoriés dans les quatre couches cartographiques, les indices topographiques du morceau permettent de dessiner un contexte particulier et par extension de visualiser les multiples interactions qui se produisent entre un enregistrement sonore et un environnement géographique. Le créateur est relié aux couches cartographiques par un système vectoriel centrifuge et centripète. Dans un sens, l'environnement influence sa création et dans l'autre sa création influence l'environnement. C'est-à-dire que pour le vecteur centrifuge, l'environnement du créateur (dont fait partie l'entité urbaine) aura une ascendance sur la création en elle-même. Cette ascendance peut provenir

5 Voir à ce sujet: Wilson 2012. 


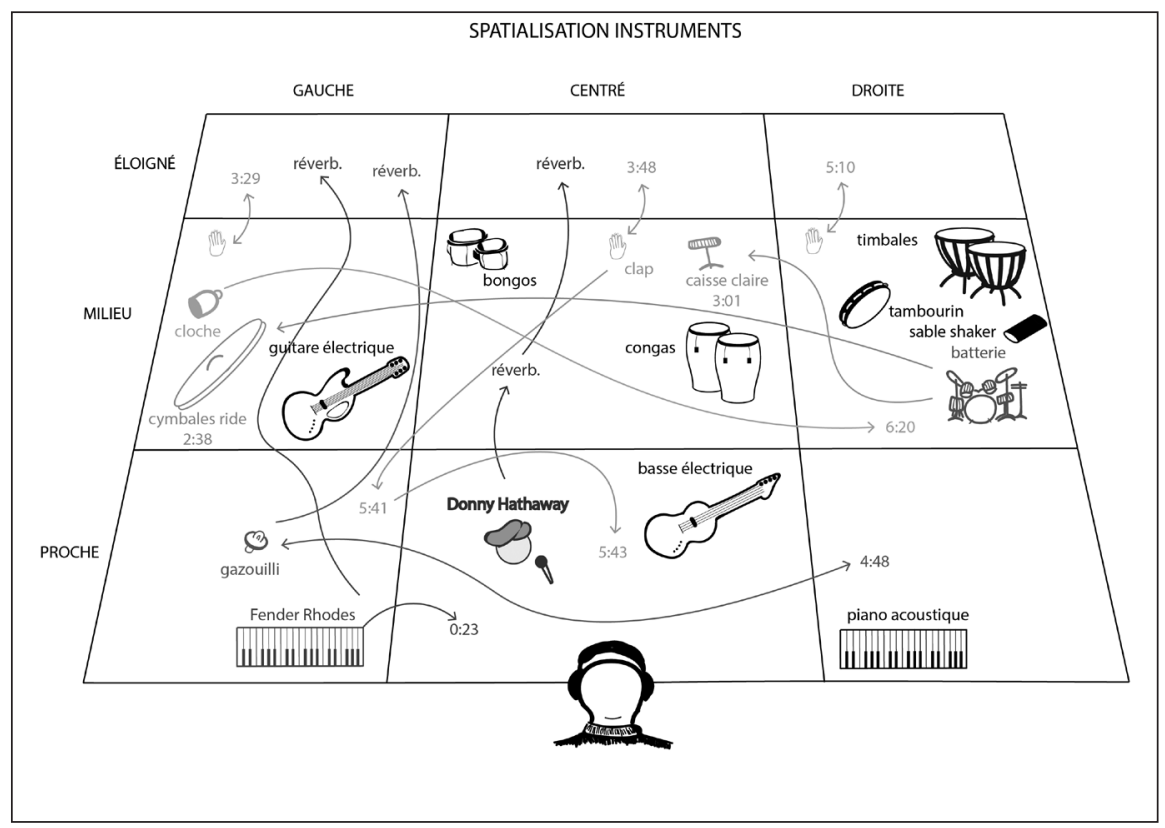

Figure 2. Parcours d'écoute de la spatialisation des instruments dans l'enregistrement de «The Ghetto» de Donny Hathaway.

de politiques propres à l'institution qui produit le morceau de musique (en imposant le format single, par exemple), au contexte économique qui impose au créateur certaines restrictions dans sa musique, ou encore à une réalité sociale (comme celle du ghetto) dépeinte dans le morceau. À l'inverse, le vecteur centrifuge observe la dynamique du créateur influençant son environnement, comme dans le cas de Donny Hathaway embellissant le ghetto avec son morceau. Dans le cas d'une cartographie sonore, deux caractéristiques sont à ajouter:

\section{Implication de l'utilisateur}

L'implication de l'utilisateur d'une carte peut être active, passive, ou les deux. Soit il suit un trajet qui lui est imposé, soit il improvise et construit sa trajectoire. Cette caractéristique oppose l'anticipation du point de vue extérieur à la sérendipité du point de vue intérieur.

\section{Type}

Enfin, la carte peut être soit dynamique, comme une carte des courants marins, soit statique comme une carte géologique de montagne. Dans les constituants de la carte, le contenu se distingue du contenant pour cette caractéristique. Cependant, dans le cas d'une cartographie sonore, il s'agira de cartes dynamiques puisqu'elles font intervenir la dimension sonique. 


\section{SPOTIFy, BruXelles ET MoNTréAL}

À titre d'exemple, nous allons appliquer cette grille d'analyse sur trois cartographies sonores bien différentes. Pour la première, il s'agit d'une spatialisation de l'information contenue dans le logiciel Spotify: Every noise at once (McDonald 2013). La première page organise tous les genres du logiciel selon un algorithme bien précis qui place la musique plus organique en bas, la plus électrique en haut, la plus atmosphérique à gauche et la plus rythmée à droite. Au bas de la page, l'auteur propose plusieurs points de vue originaux dans la même perspective, dont l'un est un répertoire de genres musicaux s'étant développé dans une ville particulière. Les villes sont listées verticalement selon le nombre de genres musicaux qui s'y développent, classés, eux horizontalement à droite du nom de la cité. La ville la plus fournie se trouve au sommet de la liste et la moins fournie au plus bas. Dans ce graphique, des villes comme Londres ou Los Angeles apparaissent considérablement plus dynamiques que d'autres villes comme Marseille, Tijuana ou Québec. Or différents paramètres d'analyses ne sont pas pris en compte comme la taille de la ville en question, et ses politiques culturelles propres. Mais voyons plus loin, le site nous propose également un répertoire de genres musicaux catégorisés par pays, dont l'organisation est à l'inverse de la page précédente. Sur cette liste, on remarque que le qualificatif patriotique devient un marqueur identitaire du pays. C'est le cas de la Pologne, qui ne propose pas de «hip-hop», mais plutôt du «hip-hop polonais».

En fait, derrière ce travail numérique titanesque se cache un reflet de la perception, ou l'espace de représentation, qu'un fragment de la population (probablement majoritairement occidental) se fait du monde musical. L'auteur ne peut se permettre de développer un regard critique puisque son projet est une spatialisation de l'information musicale du logiciel Spotify. Il est à noter que la Corée du Nord est absente du tableau. Cela ne signifie pas pour autant qu'il n'y a pas de musique dans ce pays! Le réseau est imposé à l'utilisateur qui y vagabonde de manière active comme bon lui semble, mais sans pour autant laisser de traces. La curiosité mène l'utilisateur d'un genre à un autre et d'un artiste à un autre dans un réseau cartographié qui lui est imposé. Toutefois le trajet propre à l'utilisateur/flâneur n'est jamais immortalisé par un tracé décisif. Si sa démarche est active, l'absence de trace de son passage empêche toute création empirique.

Le second exemple est celui de BNA-BBOT: Brussels Soundmap. Cette carte présente directement dans son manifeste un objectif politique engagé de rendre audible l'inaudible (Gillié et Janssen 200o), ce qui signifie mettre au-devant de la scène les dialectes, atmosphères et interprétations qui émanent d'un étouffement toujours plus constant dans une ville particulièrement hétéroclite. Cet étouffement prend place sur le plan politique, social, linguistique, urbanistique et institutionnel. Bruxelles abrite une infinité d'institutions internationales et nationales qui entraînent avec elles des règlementations particulières et des populations aux cultures bien diverses qui colorent la palette sociale de la ville cosmopolite. Sur le plan linguistique, le pays compte trois langues officielles (français, flamand et allemand) et de nombreux dialectes (dont celui de la ville 
en elle-même, le brusseleer), sans mentionner l'anglais qui simplifie la communication dans de nombreux cas. Cependant, si réactives que les autorités politiques puissent être dans un tel contexte, certains groupes sociaux se retrouvent parfois délaissés. Dans cette cartographie, les auteurs permettent aux personnes issues de ces groupes sociaux de s'adresser au monde en tant que témoins d'un moment. Comme le disent les créateurs de la cartographie sur la page explicative du site Internet: « Toute parole contient ses propres marqueurs culturels, anthropologiques, sociologiques: des ratés, des inachevés, des hésitations, des accents, des silences que la transcription ne rend généralement pas (Gillié et Janssen 2000)». Dans ce projet, la parole (et le son par extension) est considérée comme un évènement sous forme de moment historique, de la même manière que l'est le chronotope chez Bakhtin (1981).

La première page du site nous propose une série d'articles témoignant des différentes interprétations de l'environnement sonore bruxellois. Un lien nous conduit vers la véritable cartographie dans laquelle le navigateur écoute les sons de la ville, lit leurs descriptions; plus important encore, il trace une trajectoire au sein du paysage urbain. Les chemins empruntés dans cette carte théorique sont piétonniers, et par conséquent profondément individuels et personnels. Ce caractère psychologique repose sur la dimension empirique de la démarche active de l'utilisateur. Un autre lien envoie l'utilisateur vers une banque de données comprenant une multitude d'entretiens, sur différents sujets, avec les habitants de Bruxelles. L'engagement politique, social et culturel est ici évident. De plus, les différentes perceptions de l'espace urbain que l'on retrouve dans les nombreux entretiens incluent la ville de Bruxelles dans le concept «d'HyperCité» énoncé plus haut. En vagabondant sur cette cartographie sonore, nous visitons la ville de l'intérieur et les témoignages figent moment précis du passé et par conséquent, de l'histoire de la cité.

Le dernier exemple est celui de la Carte sonographique de Montréal (Stein et Stein 200o). Dans cette dernière interface, l'utilisateur écoute les ambiances sonores de la ville, lit les informations détaillées du son qui y est produit; mais la carte n'offre malheureusement pas une démarche aussi ludique que celles vues précédemment. En réalité, elle possède un horizon politique: elle illustre parfaitement l'intérêt que peut avoir une ville à posséder sa propre cartographie sonore. En effet, puisqu'il s'agit d'une représentation du territoire, elle doit convenir aux attentes de l'état, afin que celui-ci finance tout projet visant le développement d'un patrimoine culturel. En effet, la cartographie sonore contribue à l'élaboration de l'identité culturelle d'une cité: «Il est dans l'intérêt des gestionnaires des politiques culturelles de faire en sorte que les arts créatifs, commerciaux ou autres servent la cause de la politique culturelle ${ }^{6}$ (Singh 2011, 9). À partir de ce point de vue apparait le paradoxe suivant: le très engagé (comme nous l'avons vu plus haut) BNA-BBOT: Brussels Soundmap est précisément financé par les institutions culturelles de la ville de Bruxelles alors que cela ne semble pas être le cas pour la Carte sonographique de Montréal.

6 «It is in the interest of cultural-policy stakeholders to make creative arts, commercial or otherwise, serve the cause of cultural policy» (traduction par l'auteur). 
La cartographie sonore, comme d'autres types de cartographies, virevolte donc autour de cette frontière qui sépare ce qui est contrôlé par l'État, de ce qui ne l'est pas. La Carte sonographique de Montréal rend bien compte de cette dichotomie du territoire, celui de la ville de Montréal, de manière sonique, cependant aucune prise de position n'est révélée. Ce n'est pas le cas pour BNABBOT: Brussels Soundmap, qui prône son importance au sein d'un contexte contemporain particulier. La représentation du territoire par l'habitant offre une image alternative de la ville par rapport à celle, théorique, contrôlée par une quelconque autorité. De la même manière, dans Every Noise At Once, l'utilisateur n'est pas seulement libre de titiller sa curiosité musicale et de découvrir de nouveaux genres, il fait également face au reflet d'un espace de représentation du monde musical d'une certaine tranche de la population mondiale. Pourtant, l'engagement de cette cartographie sonore n'est pas manifeste. D'où la fonction-clé de la cartographie sonore qui est celle de la représentation alternative du territoire, une "contre-carte», en somme.

L'intérêt d'une cartographie sonore prend place également dans les problématiques territoriales au Canada, plus particulièrement dans le cas de la Gaspésie. Outre les querelles folkloriques qui délogent parfois la frontière entre la Gaspésie et la région du Bas-Saint-Laurent par rapport à sa position de base officielle, la Gaspésie était également auparavant un territoire usuellement foulé par les communautés Mi'kmaq et Malécites. Ces communautés se sont retranchées avec le temps pour s'établir à Listuguj (McMillan et Yellowhorn 2004), à la frontière entre le Québec et le Nouveau-Brunswick. En outre, la Gaspésie se dote d'une palette sociale variée allant d'une ancienne ville minière comme Murdochville au tourisme de luxe sur les côtes atlantiques. Sa situation géographique offre également un environnement sonore bien particulier puisque le vent fort est partie intégrante du mode de vie gaspésien. Cette diversité sociale et géographique présente un terrain idéal pour la création d'une cartographie sonore particulière se basant sur les deux derniers exemples cités plus haut. Dans un second temps, le modèle méthodologique proposé plus haut permet de déceler les indices topographiques d'un morceau de musique, qui dès lors deviendrait partie intégrante d'une cartographie sonore sur base d'un territoire géographiquement existant. Qu'il s'agisse de musique gaspésienne particulièrement ou de musique soul des années 1970 à New York, le modèle «recontextualise» le morceau et permet de le replacer sur une carte. Ainsi, "Across the $110^{\text {th }}$ street» de Bobby Womack se placerait sur la rue du même nom et «The Ghetto» de Donny Hathaway, dans un centre communautaire de Harlem, par exemple. Établir une cartographie sonore d'un ancien ghetto américain uniquement avec des morceaux du genre soul de la même époque livrerait un aspect culturel au quartier spécifique mêlant le visuel et le musical. C'est dans ce sens que mes recherches se poursuivront.

\section{Conclusion}

Pour récapituler, depuis la seconde moitié du $\mathrm{XX}^{\mathrm{e}}$ siècle, la production de contrecartes a crû considérablement, aidée par une solide fondation scientifique dans 
l'objectif commun d'une réappropriation du territoire autoritaire par un individu. L'imbrication des deux dimensions personnelle et impersonnelle intègre une ville dans le concept «d'HyperCité». Comme cas particuliers "d'HyperCité», nous avons parcouru celui de Bruxelles, de Montréal et dans le cas de Every noise at once, nous serions presque en mesure de parler d'un "HyperMonde». Les paramètres (ou modalités) d'une cartographie sonore peuvent se mesurer à l'aide d'un l'outil méthodologique basé sur les quatre couches cartographiques énoncées plus haut. Celui-ci permet également d'analyser les interactions entre un enregistrement musical et son environnement géographique, permettant ainsi de "recontextualiser» géographiquement l'enregistrement en question. À la suite d'un examen concernant ces paramètres, nous sommes en mesure de positionner une cartographie sonore particulière autour de la frontière de la neutralité.

\section{RÉFÉRENCES}

Bakhtin, Mikhail. 1981. "Forms of Time and of the Chronotope in the Novel». Dans Michael Holquist, The Dialogic Imagination - four essays by M. M. Bakhtine, 84-290. Texas: University of Texas Press. (Publ. orig. 1937).

Branch, Jordan. 2014. The Cartographic State. New York: Cambridge University Press.

Debord, Guy-Ernest. 1955. "Introduction à une critique de la géographie urbaine». Les lèvres nues, no. 6. (consulté le 07.10.2018) https://www. larevuedesressources.org/introduction-a-une-critique-de-la-geographie -urbaine, $033 \cdot \mathrm{html}$.

de Certeau, Michel. 1990. L'invention du quotidien 1. Arts de faire. Paris: Gallimard. (Publ. orig. 1980).

Deleuze, Gilles, et Félix Guattari. 1980. Capitalisme et schizophrénie 2: Mille plateaux. Paris: Les éditions de minuit.

Clark, Kenneth B. 1989. Dark Ghetto: Dilemmas of Social Power. New York: Harper \& Row. (Publ. orig. 1965).

Cooper, David. 2015. "Critical Literary Cartography: Text, Maps and a Coleridge Notebook» dans Les Roberts, Mapping cultures: Place, Practice, Performance, 29-53. Londres: Palgrave MacMillan.

Crone, Rainer, et Alexandra Von Stosch. 2008. Anish Kapoor. Munich: Prestel. Foucault, Michel. 1975. Surveiller et punir. Paris: Gallimard.

McCartney, Andra. 2010. "Ethical questions about working with soundscapes», présentation dans le cadre du WFAE international conference Ideologies and Ethics in the Uses and Abuses of Sound (19 juin). Finlande, Koly (consulté le o6.08.2018) https://soundwalkinginteractions.wordpress. com/category/presentations/.

McMillan, Alan D., et Eldon Yellowhorn. 2004. First people in Canada. Madeira Park: Douglas \& McIntyre

Lefebvre, Henri. 200o. La production de l'espace. Paris: Anthropos. (Publ. orig. 1974).

Liinamaa, Saara. 2014. «The Artist as Urban Researcher: Research, Representation and Image Relations in the City». Dans Michael Darroch, et Janine 
Marchessault, Cartographies of Places: Navigating the Urban, 92-117. Montréal: McGill-Queen's University Press.

Presner, Todd, David Shepard, et Yoh Kawano. 2014. HyperCities: Thick Mapping in the Digital Humanities. Cambridge: Harvard University Press.

Schafer, Murray R. 2010. Le Paysage Sonore: Le monde comme musique. Paris: Éditions Wildproject. (Publ. orig. 1977).

Singh, J.P. 2011. Globalized Arts: The Entertainment Economy and Cultural Identity. New York: Columbia University Press.

Spies, Werner, et Wolfgang Volz. 1980. The Running Fence Project: Christo. New York: Harry N. Abrams.

Waldock, Jacqueline. 2011. «SOUNDMAPPING: Critiques and Reflections on this New Publicly Engaging Medium». Journal of Sonic Studies, nº. Leiden: Leiden University Press (consulté le o6.08.2018) http://journal.sonicstudies.org/volo1/nro1/ao8.

Wilson, William J. 2012. The Truly Disadvantaged: The Inner City, the Underclass, and Public Policy. Chicago: University of Chicago Press. (Publ. Orig. 1987).

\section{Filmographie}

Payne, Andrew, dir. Unsung. Saison 1, épisode 3. «Unsung: Donny Hathaway ». Diffusé le 2 décembre 2008 sur TV One.

Shears, Barry. Across the 11oth street. U.S.A.: United Artists. 1972

\section{ENREGISTREMNTS}

Hathaway, Donny. A Donny Hathaway collection. Compilation. Atlantic 82092-2. 1990.

Womack, Bobby. Across the $110^{\text {th }}$ street. J.J. Johnson \& His Orchestra. Bobby Womack. Charly Records 803415123124. 2001.

\section{WEBOGRAPHIE}

(Ressources consultées le 6 août 2018)

McDonald, Glen. 2013. Every Noise At Once: http://everynoise.com/engenremap.html

Gillié, Flavien, et Séverine Janssen. 2000. BNA-BBOT: Brussels Soundmap: http://www.bna-bbot.net/Public/.

Stein, Max et Julian Stein. 200o. Carte sonographique de Montréal: http://www. montrealsoundmap.com/?lang=fr.

\section{Guvres D'ART}

Khalili, Bouchra. 2008-2011. The Mapping Journey Project. Installation audiovisuelle en couleur de 8 séquences différentes: (consulté le 06.08.2018) http://www.bouchrakhalili.com/the-mapping-journey-project/.

Kapoor, Anish. 2004. Cloud Gate. Sculpture monumentale en miroir: (consulté le 07.10.2018) http://anishkapoor.com/110/cloud-gate-2.

Christo. 1972-1976. Running Fence. Mur en Rideau: (consulté le 07.10.2018) http://christojeanneclaude.net/projects/running-fence.

Beuys, Joseph. 1974. I like America and America likes me. Performance. 


\title{
RÉSUMÉ
}

Les cartographies sonores émergent et se développent de plus en plus depuis les années 200o. À l'origine, il ne s'agissait que d'une simple représentation sonique d'un lieu, mais progressivement ces interfaces numériques ont basculées de «cartes» à "contrecartes» par l'engagement social, culturel ou politique de leurs créateurs. Outre des ambiances sonores, ces «contre-cartes» révèlent des points de vue particuliers sur l'environnement quotidien d'un individu, sans se soumettre à un contrôle d'une quelconque autorité. Cet article observe les enjeux de ces créations à travers plusieurs exemples et propose un modèle d'analyse des indices topographiques d'un enregistrement sonore.

\begin{abstract}
Primarily intended as a mere sonorous depiction of a site, these digital interfaces have now steadily switched from being "maps" to being "counter-maps" through the social, cultural or political commitment of their creators. Besides providing sonic landscapes, these "counter-maps" reveal some specific perspectives upon the daily environment of an individual, regardless of any authoritative control. This article addresses issues at stake regarding these artworks, by examining some of their tokens and providing a model for analyzing topographic markers in a sonic recording.
\end{abstract}

\section{BIOGRAPHIE}

Rainier Leloup a obtenu le grade de bachelier en histoire de l'art (orientation musicologie) à l'Université Libre de Bruxelles. En 2015, il décide alors de poursuivre ses études en musicologie à l'Université Laval de Québec. Intéressé par les musiques populaires, son sujet de mémoire aborde la relation entre le morceau «The Ghetto» (1970) du musicien soul noir américain Donny Hathaway, et les ghettos des États-Unis dans les années 1970. Durant l'année 2017-2018, il fait partie du comité étudiant de l'OICRM, et participe ainsi activement à l'organisation du colloque étudiant. En 2018, il est récipiendaire de la Mention d'Honneur au concours George Proctor (SOCAN) pour ses recherches sur les cartographies sonores. Diplômé en 2018 à la maitrise en musicologie sous la direction des professeurs Sophie Stévance et Serge Lacasse, il projette d'entamer dès Janvier 2019 un doctorat en cotutelle entre l'Université Laval et l'Université de Liège en Belgique afin de poursuivre ses recherches en géographie musicale. 